\title{
Evaluación de dos pruebas rápidas [NOW® ICT Malaria Pf/Pv y OptiMAL®] para el diagnóstico de paludismo en Tumaco, Colombia
}

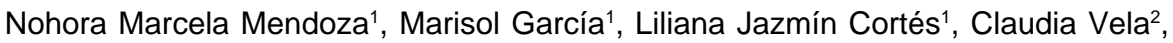 \\ Rigoberto $\mathrm{Erazo}^{2}$, Pilar Pérez², Olga Lucía Ospina ${ }^{1}$, Javier Darío Burgos ${ }^{3}$ \\ ${ }^{1}$ Grupo de Parasitología, Red Nacional de Laboratorios, Instituto Nacional de Salud, Bogotá, D.C., Colombia \\ 2 Instituto Departamental de Nariño, Pasto, Colombia \\ ${ }^{3}$ Instituto de Estudios Ambientales (IDEA), Universidad Nacional de Colombia, Bogotá, D.C., Colombia
}

Introducción. El Instituto Nacional de Salud con el fin de mejorar el acceso al diagnóstico de paludismo, evaluó dos pruebas rápidas para el diagnóstico de dicha enfermedad.

Objetivo. Evaluar la sensibilidad, especificidad y concordancia de dos pruebas inmunocromatográficas, NOW® ICT Malaria Pf/Pv y OptiMAL®, frente a la gota gruesa.

Materiales y métodos. Se llevó a cabo un estudio descriptivo de concordancia en 214 pacientes de Tumaco, captados mediante búsqueda pasiva y brigadas de atención, que presentaran, por lo menos, uno de los síntomas de la tríada clásica.

Resultados. NOW® ICT tuvo una sensibilidad general de $98,4 \%$ (intervalo de confianza de 95\% (IC95\%): 90,3-99,9), especificidad general de 98,0\% (IC95\%: 93,9-99,5). Para Plasmodium falciparum la sensibilidad fue de 98,2\% (IC95\%: 89,4-99,9) especificidad de 98,1\% (IC95\%: 94,1-99,5). Esta sensibilidad disminuyó a 80\% en el rango de 200-4.000 parásitos/ $\mu$ l. Los valores de sensibilidad y especificidad de NOW® ICT para Plasmodium vivax fueron del $100 \%$ y no se afectó la sensibilidad en los rangos de parasitemias establecidos.

OptiMAL® tuvo una sensibilidad general de $95,2 \%$ (IC95\%: 85,8-98,8) y especificidad general de 99,3\% (IC95\%: 95,8-100,0). Para P. falciparum OptiMAL $\circledast$ tuvo una sensibilidad de $94,7 \%$ (IC95\%: 84,5-98,6) y especificidad de 99,4\% (IC95\%: 96,0-100,0). La sensibilidad disminuyó a $60 \%$ en el rango de de $200-4.000$ parásitos $/ \mu l$.

La sensibilidad de OptiMAL® para P. vivax fue $66,7 \%$ (IC95\%: 24,1-94,0), pero disminuyó a $50 \%$ en el rango de 300-2.500 parásitos $/ \mu$ l.

Conclusiones. Se obtuvieron buenos resultados en la sensibilidad y la especificidad para ambas pruebas, pero superiores para NOW®ICT.

Palabras clave: malaria/diagnóstico, sensibilidad y especificidad, parasitemia, Plasmodium vivax, Plasmodium falciparum, Colombia.

Evaluation of two rapid diagnostic tests, NOW ${ }$ ICT Malaria Pf/Pv and OptiMAL $\circledast$, for diagnosis of malaria

Introduction. To increase the accessibility of malaria diagnosis, the Instituto Nacional de Salud de Colombia undertook a field trial to evaluate the sensitivity and specificity of two rapid diagnostic tests.

Objective. The sensitivity, specificity and concordance was compared for two rapid diagnostic tests for malaria, NOW® ICT Malaria Pf/Pv and OptiMAL $\circledast$,

Materials and methods. A descriptive and concordance study was performed with 214 patients in the southwestern coastal city of Tumaco, Colombia, each of whom presented at least one of the symptoms of the classical malaria triad. Two strategies were applied for patient recruitmentone by passive search and a second through local health brigades.

Results. NOW® ICT showed a general sensitivity of $98.4 \%$ (95\%Cl: $90.3-99.9)$, and a general specificity of $98.0 \%$ (95\% Cl: $93.9-99.5)$. For Plasmodium falciparum, the sensitivity was $98.2 \%$ (95\% $\mathrm{Cl}: 89.4-99.9)$ and the specificity $98.1 \%(95 \% \mathrm{Cl}$ : $94.1-99.5)$. The sensitivity was lower 
(80.0\%) when parasitemia ranged from 200 to 4,000 parasites/ $\mu$ l. The sensitivity and specificity of the NOW® ICT for $P$. vivax malaria were $100 \%$. The sensitivity for this test was not affected for the established ranges of parasitemia for $P$. vivax. The overall figures for OptiMAL® were 95.2\% (95\%Cl: 85.8-98.8) sensitivity and 99.3\% (95\%Cl: 95.8-100.0) specificity. For P. falciparum malaria OptiMAL® showed $94.7 \%(95 \% \mathrm{Cl}$ : $84.5-98.6)$ sensitivity and $99.4 \%(95 \% \mathrm{Cl}: 96.0-$ $100.0)$ specificity. The sensitivity was lower $(60.0 \%)$ when samples with $200-4,000$ parasites/ $\mu$ l were tested. For $P$. vivax, OptiMAL® presented a 66.7\% (95\%Cl: 24.1-94.0) sensitivity, which diminished to $50 \%$ with a parasitemia between $300-2.500$ parasites/ $\mu \mathrm{L}$.

Conclusions. Good results for sensitivity and specificity were obtained for malaria diagnosis using NOW® ICT and OptiMAL®, with NOW®ICT showing higher sensitivity and specificity values than OptiMAL®.

Key words: malaria/diagnosis, sensitivity and specificity, parasitemia, Plasmodium vivax, Plasmodium falciparum, Colombia.

De acuerdo con la evaluación anual del programa de la red de diagnóstico de paludismo liderada por el Instituto Nacional de Salud, se conoce que ésta es atendida en las zonas rurales de Colombia por microscopistas y en las urbanas por bacteriólogos que, aunque están integrados a la red, son cambiados con alta frecuencia por personal nuevo y sin experiencia, lo cual conlleva a una disminución de la capacidad técnica de la red.

Debido a la situación actual de conflicto del país, existen zonas en las cuales confluyen diferentes factores que favorecen la dispersión y transmisión del paludismo. Actualmente, las poblaciones flotantes que se desplazan a causa de la violencia y en busca de nuevas oportunidades laborales pueden llegar a diferentes regiones del país deteriorando la situación epidemiológica de algunos municipios receptivos para la enfermedad.

En zonas rurales endémicas para malaria, en donde la red de diagnóstico no cuenta con una cobertura adecuada, los pacientes deben acudir en busca de diagnóstico a lugares distantes de su sitio de vivienda o de trabajo. La tendencia más común de estos pacientes es optar por la automedicación, administrándose esquemas de tratamiento incorrectos que inducen el aumento de la resistencia de los parásitos a los medicamentos y de la morbimortalidad en el país.

\footnotetext{
Correspondencia:

Nohora Marcela Mendoza, Grupo de Parasitología, Instituto Nacional de Salud, Avenida Calle 26 № 51-20.

Fax: 220 7700, extenión 205

nmendoza@ins.gov.co marcemendoza@etb.net.co

Recibido: 20/04/07; aceptado: 03/09/07
}

Con el propósito de brindar alternativas en el diagnóstico de malaria, el Instituto Nacional de Salud, a través de la red de laboratorios y acogiéndose a las recomendaciones de la estrategia mundial de "Hacer retroceder la malaria" (Roll back malaria), estableció una línea de trabajo de ampliación de la cobertura del diagnóstico y tratamiento del paludismo, en la cual una de las actividades consistió en evaluar la capacidad diagnóstica de las pruebas rápidas.

En el año 2000, se evaluó la prueba rápida OptiMAL $\AA$ bajo condiciones de epidemia en La Guajira, Colombia, con resultados muy satisfactorios: la sensibilidad general de la prueba fue de $98,7 \%$ y su especificidad general de $99,3 \%$. La sensibilidad para Plasmodium falciparum fue $98,1 \%$ y la especificidad $76,9 \%$. Para Plasmodium vivax la sensibilidad fue de $90,9 \%$ mientas que la especificidad fue de $100,0 \%$ (1).

Este trabajo pretende ampliar la información obtenida en el estudio de La Guajira, enfocando las acciones del estudio en Tumaco, municipio de la costa del Pacífico de Colombia, zona de alta transmisión de malaria por $P$. falciparum (2), y evaluando dos pruebas de diagnóstico inmunocromatográficas NOW® ICT y OptiMAL $\AA$ frente a la gota gruesa.

Las pruebas rápidas inmunocromatográficas se fundamentan en la detección de antígenos parasitarios (proteínas), que son liberados mediante lisis celular y se unen al conjugado marcado con oro coloidal. El complejo antígeno- anticuerpo migra a lo largo de la nitrocelulosa y es anclado por anticuerpos monoclonales específicos ubicados 
sobre la misma. En estos sitios predeterminados se observa una línea de color violeta en caso de haber reacción positiva, es decir, cuando se encuentra presente el antígeno específico (3).

La prueba NOW® ICT detecta una proteína hidrosoluble, producida por trofozoítos y gametocitos maduros, denominada proteína II rica en histidina (HRP-II), específica para el diagnóstico de $P$. falciparum. Además, detecta el antígeno panmalárico, aldolasa, expresado por estadios sanguíneos de las cuatro especies de Plasmodium que causan el paludismo en humanos (3-6). Esta prueba permite diferenciar entre paludismo por $P$. falciparum y paludismo ocasionado por una especie diferente a $P$. falciparum.

Por otra parte, el OptiMAL ${ }^{\circledR}$ detecta la enzima parasitaria lactato deshidrogenasa (pLDH), la cual es producida por estadios asexuados y sexuados de las cuatro especies que causan malaria humana. La prueba cuenta con un anticuerpo monoclonal específico contra la isoenzima pLDH de $P$. falciparum y otro contra la pLDH común para las cuatro especies, lo que permite diferenciar paludismo por $P$. falciparum o por una especie diferente a ésta (3).

\section{Materiales y métodos}

\section{Tipo de estudio}

Se llevó a cabo un estudio descriptivo y de concordancia de dos pruebas inmunocromatográficas para el diagnóstico de paludismo comparadas con la gota gruesa.

\section{Lugar de estudio}

El estudio se realizó en el municipio de Tumaco, en el departamento de Nariño, Colombia, durante las semanas epidemiológicas 16 y 17 del 2002.

\section{Tamaño de la muestra}

Se calculó una muestra de 204 casos probables de paludismo $(p=0,05)$, teniendo en cuenta que la eficacia del NOW® ICT era de $85 \% \pm 5 \%$ y del OptiMAL ${ }^{\circledR}$ era de $90 \% \pm 5 \%$ (7).

\section{Población de referencia}

Estuvo integrada por los pacientes que presentaron, por lo menos, uno de los síntomas de la tríada clásica de paludismo, residentes del municipio de Tumaco, Nariño, que acudieron para diagnóstico de malaria al Laboratorio de Patologías Tropicales, y los pacientes sintomáticos captados por la brigada de atención en las veredas del municipio de Tumaco.

\section{Estrategias de atención}

El primer grupo se atendió mediante búsqueda pasiva y el segundo mediante brigadas de atención, estrategia semejante a la búsqueda activa.

\section{Criterios de inclusión}

Para el estudio se definió como caso probable de paludismo a todo paciente procedente o residente de zona endémica y que tuviera, por lo menos, uno de los síntomas de la tríada clásica de paludismo (fiebre, escalofrío y sudoración).

\section{Protocolo}

En el Laboratorio de Patologías Tropicales se atendieron los pacientes de manera rutinaria y después de la toma de muestra por punción capilar fueron remitidos al grupo de investigación en donde se siguió el protocolo del estudio.

En las brigadas de atención se procedió directamente con el siguiente protocolo.

1. Se aplicó la encuesta epidemiológica y se diligenció el consentimiento informado de manera individual.

2. A todas las personas que cumplieron con la definición de caso probable y, además, aceptaron voluntariamente participar en el estudio mediante su consentimiento informado, se les tomó una muestra de sangre total de $4 \mathrm{ml}$ en tubo al vacío con EDTA para el montaje simultáneo de las dos pruebas rápidas y para la elaboración de dos láminas para gota gruesa (método de referencia).

3. OptiMAL®: se tomó la sangre total con la pipeta plástica provista en el estuche de diagnóstico; la sangre se dejó ascender hasta el aforo de la misma. Se respetaron los tiempos de montaje según lo descrito por el fabricante. Se tomaron las siguientes precauciones: en los lugares en donde se apreciaba que el ascenso por capilaridad se veía interrumpido por el secado de la muestra, dada la elevada 
temperatura del medio ambiente, se procedió a adicionar una gota más de solución tampón para completar el corrido de la muestra y las cuatro gotas de solución tampón de lavado se adicionaron en el pozo solamente cuando se completó el corrido del lisado de sangre en el papel de nitrocelulosa.

4. Además, en los casos en que la solución tampón de lavado no corrió adecuadamente se procedió a mover la tirilla con suavidad dentro de la solución tampón y de no corregirse el problema, se adicionaba una gota más de la misma solución al pozo de lavado.

5. NOW® ICT: se tomó la sangre total con el capilar de vidrio suministrado en el estuche de diagnóstico, teniendo la precaución de soplar el capilar en los casos en que se veía obstruido con el EDTA, ya que el líquido presente impedía el ascenso de la sangre por capilaridad. Dentro de las tarjetas, se adicionó la sangre en la almohadilla lila hasta que la misma dejara de absorber la sangre o en su defecto hasta observar que la sangre rebozara el área lila. El procedimiento de montaje se siguió de acuerdo con las instrucciones que acompañan el estuche de diagnóstico. Se hizo especial énfasis en el cumplimiento del tiempo de lavado (10 minutos). Además, se tomó la precaución de mantener los capilares almacenados en temperatura de refrigeración la noche anterior para evitar que el anticoagulante se secara lo cual se evidenció por la formación de un precipitado de sal en el interior del capilar que obstruía el ascenso de la sangre.

6. Gota gruesa: se elaboraron dos láminas por paciente, las cuales fueron debidamente codificadas.

7. La lectura de las tirillas de las dos pruebas se realizó tan pronto como cumplieron el tiempo de lavado. En el caso de OptiMALß, se dejó lavar dos minutos adicionales cuando la tirilla no se observaba limpia. Los criterios de lectura que se tuvieron en cuenta para OptiMAL® fueron aquéllos sugeridos en la intervención de la epidemia en La Guajira (1) y para NOWß ICT fueron los sugeridos por el fabricante; sin embargo, siempre se interpretó como lectura positiva cuando se presentó reacción en la línea control y una banda de reacción violeta de cualquier intensidad en las posiciones de los monoclonales contra los antígenos parasitarios. Además, cuando se presentó reacción en la banda control y en la específica para la HRP-II se determinó positividad para $P$. falciparum aun cuando no existiera reacción en el antígeno común. Las tirillas fueron conservadas para realizar posteriormente control de calidad al $100 \%$ del material en el Instituto Nacional de Salud por personal capacitado.

8. Se entregaron los resultados de los exámenes por escrito a todos los pacientes que acudieron al diagnóstico y se suministraron los esquemas de tratamiento de acuerdo con la normatividad vigente del Ministerio de la Protección Social. En el Laboratorio de Patologías Tropicales el tratamiento se basó en el resultado de la gota gruesa realizada por el microscopista del puesto. Esta lectura la realizó personal independiente al estudio y guardando confidencialidad de los resultados con el fin de evitar introducir sesgos en el estudio de pruebas rápidas.

En las brigadas de salud el tratamiento se basó en el diagnóstico obtenido mediante las pruebas rápidas, cuando, por lo menos, una de las pruebas tenía resultado positivo.

9. Se coloreó una lámina por paciente con la tinción de Romanowsky modificado en el Laboratorio de Patologías Tropicales al finalizar la jornada de trabajo. Todas las láminas fueron procesadas y coloreadas con protocolos estandarizados por el Instituto Nacional de Salud. La segunda lámina de gota gruesa se descartó cuando se verificó el cumplimiento de los requisitos de calidad de la primera lámina.

Se priorizó la lectura de las láminas en las que el personal de salud tenía alguna inquietud y se deseaba rectificar o corregir la medida tomada en campo, esta acción fue tomada para cinco pacientes.

10. El total de las láminas fueron leídas posteriormente por dos lectores experimentados, 
de manera independiente y guardando confidencialidad. Cuando existió discordancia entre el primer y el segundo lector se acudió a un tercer lector experimentado. Los microscopistas desconocían el diagnóstico de las diferentes pruebas rápidas. Para el recuento parasitario se empleó la constante de 8.000 leucocitos/ $\mu \mathrm{l}$ y el número de parásitos se estimó en 100 glóbulos blancos. Cuando se encontraron muestras con un solo parásito en una de las gotas gruesas, se examinó por completo la segunda gota gruesa, teniendo en cuenta el número total de leucocitos presentes en la lámina. En altas parasitemias se empleó la constante de 500 parásitos en el número de glóbulos blancos observados.

11. La mecánica para realizar el control de calidad de las pruebas rápidas fue igual que para las gotas gruesas. Sin embargo, para el control de calidad del NOW-ICT se tuvo la precaución de dar por negativa cualquier reacción francamente débil que se presentara en la banda en donde se ubica la HRP-Il ya que esta banda de reacción en algunos casos puede presentarse como un falso positivo débil después de los 10 minutos de lavado.

\section{Análisis estadísticos}

Se hicieron análisis estadísticos y demográficos usando el programa de Statgraphics plus 4.0. Se utilizó el programa de Epilnfo versión 6.0 para calcular la sensibilidad, la especificidad, los valores pronósticos positivo y negativo para cada una de las pruebas rápidas empleadas; se trabajó con un intervalo de confianza del $95 \%$.

Además, se calcularon los índices kappa de concordancia, con sus intervalos de confianza de $95 \%$, con el fin de evaluar si las pruebas producían resultados similares cuando se aplicaban a las mismas muestras en forma simultánea o si no lo hacían.

Por otra parte, se utilizó el programa de EPIDAT Versión 3.0 para el cálculo de razón de verosimilitud o likelihood ratio.

Se estimaron intervalos de clase para las parasitemias mediante el algoritmo de Sturgess.

\section{Aspectos éticos}

El presente trabajo fue sometido a estudio y aprobación por el Comité de Ética del Instituto Nacional de Salud, cumpliendo estrictamente con lo estipulado en la Resolución No. 008430 de 1993 del Ministerio de Salud, por la cual se fijaron las normas éticas para la investigación en salud. De acuerdo con esta resolución, el estudio fue considerado de riesgo mínimo pues se obtuvo una muestra de sangre venosa de $4 \mathrm{ml}$ tomada por personal experto. Se explicó a los pacientes el propósito del estudio y los beneficios y riesgos de participar en él. Aquellos pacientes que aceptaron voluntariamente participar en el trabajo, firmaron el formato de consentimiento informado.

\section{Resultados}

De los 214 pacientes incluidos en el estudio, 135 $(63,1 \%)$ fueron hombres. La distribución por grupos de edad fue la siguiente: $0,5 \%$ eran menores de un año, 3,3\% tenían entre 1 y 4 años, 30,8\% entre 5 y 14 años, $52,3 \%$ entre 15 y $44,8,9 \%$ entre 45 y 59 años de edad y $4,2 \%$ eran mayores de 60 años.

La frecuencia de síntomas clínicos en los pacientes fue: fiebre, 202 (94,4\%); escalofríos, $162(75,7 \%)$; sudoración, 120 (88,8\%), y cefalea $190(88,8 \%)$. Otros síntomas clínicos analizados se encuentran en el cuadro 1.

Con relación a la presencia de episodios anteriores de paludismo, 86 pacientes $(40,2 \%)$ sufrieron malaria previamente y $128(59,8 \%)$ no presentaron la enfermedad anteriormente. El 95\% de los pacientes con episodios anteriores de malaria los presentaron en un tiempo mayor o igual a 30 días antes de la toma de la muestra.

De los 214 pacientes clasificados como casos probables de paludismo, 87 (40,7\%) acudieron al puesto de diagnóstico fijo ubicado en Patologías Tropicales y 127 (59,3\%) fueron diagnosticados a través del puesto de diagnóstico móvil que visitaba las veredas. Del total de pacientes, 63 (29\%) fueron positivos y 151 (71\%) fueron negativos por gota gruesa (cuadro 2).

El estuche de diagnóstico NOW® ICT tuvo una sensibilidad general de 98,4\% (IC95\%: 90,3-99,9), y una especificidad general de $98,0 \%$ (IC95\%: 
Cuadro 1. Frecuencia de síntomas en los pacientes atendidos.

\begin{tabular}{lrl}
\hline Síntoma & \multicolumn{2}{c}{$\begin{array}{c}\text { Frecuencia } \\
\text { n }(\%)\end{array}$} \\
\hline Fiebre & 202 & $(94,39)$ \\
Escalofrío & 162 & $(75,70)$ \\
Sudoración & 120 & $(56,07)$ \\
Cefalea & $190(88,78)$ \\
Artralgias & $118(55,14)$ \\
Mialgias & $39(18,22)$ \\
Náuseas & $69(32,24)$ \\
Vómito & $39(18,22)$ \\
Diarrea & $38(17,75)$ \\
\hline
\end{tabular}

$93,9-99,5)$. Se obtuvo un valor pronóstico positivo de $95,4 \%$ (IC95\%: 86,2-98,8) y un valor pronóstico negativo de 99,3\% (IC95\%: 95,8-100,0). El índice kappa fue de 0,955 (IC95\%: 0,822-1,088; $\mathrm{p}<0,001)$. La razón de verosimilitud positiva fue de 49,53 (IC95\%: 49,20-49,87) y la negativa de 0,02 (IC95\%: 0,02-0,02).

Para $P$. falciparum NOW® ICT tuvo una sensibilidad de 98,2\% (IC95\%: 89,4-99,9) y especificidad de 98,1\% (IC95\%: 94,1-99,5). Los valores pronósticos positivo y negativo fueron de 94,9\% (IC95\%: 84,9-98,7) y 99,4\% (IC95\%: 95,9100,0), respectivamente. El índice kappa fue de 0,953 (IC95\%: 0,820-1,086; $p<0,001$ ), la razón de verisililitud positiva de 51,42 (IC95\%: 51,0751,77 ) y la negativa de 0,02 (IC95\%: 0,02-0,02).

Los valores de sensibilidad y especificidad de NOW® ICT para $P$. vivax fueron de $100,0 \%$ (IC95\%: 51,7-100,0) y 100,0\% (IC95\%: 97,7$100,0)$, respectivamente. Los valores pronósticos positivo y negativo fueron de 100,0\% (IC95\%:
$51,7-100,0)$ y $100,0 \%$ (IC95\%: $97,7-100,0$ ), respectivamente. El índice kappa fue de 1,000 (IC95\%: 0,867-1,133; $p<0,001$ ); las razones de verisililitud positiva y negativa fueron indeterminadas.

La prueba OptiMAL $\circledast$ tuvo una sensibilidad general de $95,2 \%$ (IC95\%: 85,8-98,8) y una especificidad general de 99,3\% (IC95\%: 95,8-100,0). Los valores pronósticos positivo y negativo fueron de 98,4\% (IC95\%: 90,0-99,9) y 98,0\% (IC95\%: 93,9$99,5)$, respectivamente. El índice kappa fue de 0,954 (IC95\%: 0,821-1,088; $p<0,001$ ); la razón de verisililitud positiva fue de 143,81 (IC95\%: 142,11145,53 ) y la negativa de 0,05 (IC95\%: 0,05-0,05).

La sensibilidad de OptiMAL® para $P$. falciparum fue de 94,7\% (IC95\%: 84,5-98,6), la especificidad de 99,4\% (IC95\%: 96,0-100,0), el valor pronóstico positivo de $98,2 \%$ (IC95\%: 89,0-99,9) y el valor pronóstico negativo de 98,1\% (IC95\%: 94,2-99,5). El índice kappa fue de 0,951 (IC95\%: 0,818-1,084; $\mathrm{p}<0,001$ ); la razón de verisililitud positiva fue de 148,74 (IC95\%: 146,98-150,51) y la negativa de 0,05 (IC95\% 0,05-0,05).

La sensibilidad de OptiMAL® para $P$. vivax fue de 66,7\% (IC95\%: 24,1-94,0), la especificidad de 99,0\% (IC95\%: 96,2-99,8), los valores pronósticos positivo y negativo fueron de 66,7\% (IC95\%: 24,1 94,0 ) y $99,0 \%$ (IC95\%: 96,2-99,8), respectivamente. El índice kappa fue de 0,657(IC95\%: 0,524-0,790; $p<0,001$ ); la razón de verisililitud positiva fue de 69,33 (IC95\% 68,71-69,96) y la negativa de 0,34 (IC95\% 0,33-0,34).

Para $P$. falciparum se obtuvieron los siguientes rangos de parasitemia: $<200,200-4000,4001-7000$,

Cuadro 2. Comparación de NOW® ICT y OptiMAL® frente a la gota gruesa.

\begin{tabular}{|c|c|c|c|c|c|c|}
\hline \multirow{3}{*}{$\begin{array}{l}\text { Pruebas } \\
\text { NOW®-ICT }\end{array}$} & & & \multicolumn{4}{|c|}{ Gota gruesa } \\
\hline & \multicolumn{2}{|c|}{ Resultados } & & \multicolumn{2}{|c|}{ Positivo } & \multirow[t]{2}{*}{ Tota } \\
\hline & & & Negativo & P. falciparum & P. vivax & \\
\hline & & Negativo & 148 & 1 & 0 & 149 \\
\hline & Positivo & P. falciparum & 3 & 56 & 0 & 59 \\
\hline & & $P$. vivax & 0 & 0 & 6 & 6 \\
\hline & & Total & 151 & 57 & 6 & 214 \\
\hline \multirow[t]{4}{*}{ OptiMAL® } & & Negativo & 150 & 2 & 1 & 153 \\
\hline & Positivo & P. falciparum & 0 & 54 & 1 & 55 \\
\hline & & $P$. vivax & 1 & 1 & 4 & 6 \\
\hline & & Total & 151 & 57 & 6 & 214 \\
\hline
\end{tabular}


7001-10.000 y >10.000. En el rango inferior se encontró una sensibilidad del $80 \%$ para NOW® ICT y del $60 \%$ para OptiMAL®. Para los otros rangos la sensibilidad fue del $100 \%$ (cuadro 3 ).

Para $P$. vivax se obtuvieron los siguientes rangos de parasitemia: 300-2500 (cuatro muestras) y 25015500 (dos muestras), encontrándose en el rango inferior una sensibilidad del $100 \%$ para NOW® ICT y del $50 \%$ para OptiMAL $\AA$. En el rango superior la sensibilidad fue del $100 \%$ para los dos métodos.

Los falsos negativos de las pruebas rápidas fueron observados en los siguientes casos: una muestra positiva para $P$. falciparum con una parasitemia de 6,4 parásitos/ $\mu \mathrm{l}$, no detectada por ninguna de las pruebas rápidas; una muestra positiva para P. falciparum con parasitemia de 25,6 parásitos/ $\mu$ detectada por NOW ${ }^{\circledR}$ ICT pero no por OptiMAL $\AA$, y una muestra positiva para $P$. vivax con una parasitemia de 1.680 parásitos/ $\mu$ l detectada por NOW® ICT y no por OptiMAL®.

En lo que respecta a los falsos positivos, se encontró que para NOW® ICT tres muestras que fueron negativas por gota gruesa dieron un resultado positivo para $P$. falciparum, mientras que para Optimal® se encontró una muestra con resultado falso positivo para $P$. vivax.

Por otra parte, se encontraron discordancias de especie en una muestra positiva para $P$. falciparum con una parasitemia de 242 parásitos/ $\mu \mathrm{l}$, que fue identificada correctamente por NOW® ICT, pero no por OptiMAL® debido a que su resultado fue positivo para $P$. vivax; además, se encontró una muestra positiva para $P$. vivax con

Cuadro 3. Valores de sensibilidad para NOW® ICT y OptiMAL ${ }^{\circledR}$ encontrados en los rangos de parasitemias establecidos en las muestras de los pacientes positivas para $P$. falciparum.

\begin{tabular}{|c|c|c|c|}
\hline \multicolumn{2}{|c|}{ P. falciparum } & \multicolumn{2}{|c|}{ Sensibilidad } \\
\hline $\begin{array}{l}\text { Rangos de } \\
\text { parasitemias }\end{array}$ & Muestras & $\begin{array}{r}\text { NOW® ICT } \\
\%\end{array}$ & OptiMAL $\circledast$ \\
\hline$<200$ & 5 & 80 & 60 \\
\hline $200-4.000$ & 24 & 100 & 100 \\
\hline $4.001-7.000$ & 7 & 100 & 100 \\
\hline $7.001-10.000$ & 3 & 100 & 100 \\
\hline$>10.000$ & 18 & 100 & 100 \\
\hline
\end{tabular}

una parasitemia de 480 parásitos $/ \mu$, que fue identificada de manera adecuada por NOW® ICT pero no así por OptiMAL® ya que fue positiva para $P$. falciparum.

Finalmente, de las seis muestras que presentaron dificultades en la lectura del diagnóstico rápido por presentar reacciones muy tenues, se encontró el mismo número de muestras con dificultades tanto para NOW® ICT como para OptiMAL ${ }^{\circledR}$, pero sólo dos muestras fueron comunes entre sí.

\section{Discusión}

Al igual que otros estudios en Suramérica $(8,9)$, se encontraron altos valores de sensibilidad y especificidad para las pruebas rápidas. La sensibilidad y especificidad para paludismo tanto general como específica para $P$. falciparum de las dos pruebas rápidas son consideradas adecuadas para el diagnóstico parasitario ya que los valores encontrados fueron superiores al 95\% $(8,9)$.

Los índices de concordancia encontrados son adecuados por ser iguales o superiores a 0,95, excepto para la prueba Optimal® en la detección de P. vivax.

A diferencia del estudio realizado por van Den Broek et al. en el 2006, en el que encuentran una alta sensibilidad y especificidad para Optimal® en la detección de $P$. vivax (10), en el presente trabajo la sensibilidad encontrada para dicha prueba se considera pobre, mientras que $\mathrm{NOW}{ }^{\circledR}$ ICT obtuvo un excelente desempeño del NOW ${ }^{\circledR}$ ICT. Lo anterior pudo ocurrir por la baja incidencia de casos de paludismo por $P$. vivax en el municipio de Tumaco; de igual manera es probable que este resultado se deba a la diferencia del antígeno blanco para cada prueba. Esta baja sensibilidad del OptiMAL® no se obtuvo en un estudio anterior realizado en Colombia en el departamento de La Guajira (1).

Aunque existen resultados satisfactorios del desempeño de estas dos pruebas rápidas para el diagnóstico de paludismo en la literatura $(6,9,11$ 13), en el presente estudio se obtuvieron valores superiores en algunos casos, tal vez debido a los aspectos epidemiológicos o a factores técnicos tenidos en cuenta en el almacenamiento, transporte, capacitación, interpretación y 
acompañamiento al personal involucrado en el desarrollo del estudio.

Al igual que en otros estudios reportados en la literatura científica, se encontró que la sensibilidad de las pruebas rápidas disminuye directamente ante parasitemias bajas (10,14-18); sin embargo se encontró que NOW ${ }^{\circledR}$ ICT mostró mayor sensibilidad en los rangos de parasitemias inferiores para las dos especies parasitarias.

Del total de muestras positivas para $P$. falciparum, solamente una contaba con gametocitos en una concentración de 80 gametocitos/ $\mu$; dicho paciente presentó la tríada clásica de síntomas y refirió nunca antes haber tenido malaria. En este caso, las dos pruebas rápidas tuvieron resultado positivo y debido a que la conducta a seguir con el paciente se fundamentó en el resultado de las pruebas rápidas, al paciente le fue suministrado el tratamiento específico. En este caso se pueden plantear algunas hipótesis tales como:

a. La existencia de inconsistencias en la información suministrada por el paciente. Sin embargo, el paciente pudo haber tenido paludismo anteriormente y pudo haber sido tratado correctamente en algún puesto de diagnóstico y, por lo tanto, dichos gametocitos no representaban peligro en la transmisión de paludismo en la zona y los síntomas presentes en él fueron ocasionados por otra enfermedad febril, por lo que este tratamiento no fue suministrado racionalmente. Sin embargo, en las condiciones de campo y sin diagnóstico microscópico, ésta fue la mejor opción.

b. El paciente pudo haber tenido paludismo anteriormente y pudo no haber sido tratado correctamente con respecto al antipalúdico con acción gametocitocida, bien porque el tratamiento hubiera sido suministrado de manera incompleta (sin primaquina) o bien por que el paciente hubiera recurrido a una automedicación inadecuada, motivo por el cual dichos gametocitos representaban un riesgo de transmisión de la enfermedad para la comunidad.

c. Además, se puede pensar que el paciente pudo haber tenido una infección palúdica que fue tratada con un antipalúdico con acción esquizonticida, el cual disminuyó la parasitemia asexual en un grado tal que en el momento de la toma de la muestra no era detectable por la gota gruesa, aunque sí fue posible encontrar gametocitos en circulación. Si a este hallazgo se le suman los síntomas del paciente, se pudiera plantear la posibilidad de que el paciente fue tratado con un esquema incorrecto y que tal vez con la ayuda de un correcto seguimiento y con el examen microscópico se hubiera podido establecer una posible falla terapéutica.

De todas formas, esta situación solamente se presentó en uno de los 57 pacientes positivos para $P$. falciparum lo que representa $1,7 \%$, es decir, que al suministrar este tratamiento completo existió un beneficio directo en la comunidad al evitar la transmisión de la enfermedad y, posiblemente, un beneficio de carácter individual en el paciente.

Debido a la alta prevalencia de $P$. falciparum en el área de estudio, se esperaba encontrar un mayor porcentaje de falsos positivos para la identificación de $P$. falciparum sobre todo para NOW® ICT por el tipo de antígeno que utiliza para la detección de esta especie (HRP-II), el cual es conocido por su alta estabilidad y mayor duración en circulación después del tratamiento $(19,20)$.

Es importante continuar realzando la utilidad de las pruebas rápidas para el diagnóstico de paludismo, manteniendo las condiciones de temperatura y humedad que establece el fabricante. Cuando dichas condiciones no se puedan garantizar es importante establecer y mantener una cadena de frío durante el transporte y tiempos de intervalos en los cuales no se esté trabajando con los estuches de pruebas rápidas, con el fin de contar con reactivos en buenas condiciones.

Un escenario en donde las pruebas rápidas son una herramienta de diagnóstico importante es en la fase previa al fortalecimiento de la red de diagnóstico de microscopía de paludismo, por lo que debe considerarse como una estrategia transitoria mientas se concretan acciones para mejorar la accesibilidad de la red o para hacer mantenimiento 
o reposición de los equipos de la misma, casos en los cuales es posible programar brigadas de atención a la población descubierta o es posible implementar un puesto de diagnóstico de pruebas rápidas para malaria que cuente con las condiciones para preservar los reactivos en refrigeración.

Con el uso adecuado de este tipo de diagnóstico parasitario es posible racionalizar los medicamentos antipalúdicos a los casos confirmados y no hacer uso indiscriminado de ellos en pacientes febriles con clínica poco específica.

Otro escenario en donde resulta ser útil la prueba rápida es cuando no se cuenta con personal idóneo o con gran experiencia para realizar el diagnóstico microscópico de malaria. En estas circunstancias los entes territoriales pueden programar recursos para establecer un pequeño depósito de pruebas rápidas mientras se logra la calidad requerida del diagnóstico microscópico.

Sin embargo, siempre será mucho más fácil y económico insistir en mantener y fortalecer la red de microscopía de malaria en lo referente a capacitación, actualización, control de calidad, dotación y mantenimiento preventivo y correctivo de los equipos.

\section{Agradecimientos}

Los autores agradecen el apoyo del Instituto Departamental de Salud de Nariño, a todos los pacientes del municipio de Tumaco y al personal técnico de malaria que participó en este trabajo.

De igual manera, los autores presentan sus agradecimientos al bacteriólogo Luis Gonzalo Álvarez Sánchez, docente investigador de la Facultad de Odontología de la Universidad CES quien colaboró con aportes estadísticos.

\section{Conflicto de intereses}

Los autores manifiestan no tener ningún nexo con las entidades comerciales que venden o distribuyen las pruebas rápidas para el diagnóstico rápido de malaria, como tampoco tienen conflicto de interés ni compromiso con estas entidades.

\section{Financiación}

Instituto Nacional de Salud con recursos asignados por el Ministerio de la Protección Social.

\section{Referencia}

1. Mendoza NM, Montoya R, García M, Padilla JC, Bruzón LO, Mendoza E, et al. Evaluación de campo de una prueba rápida para el diagnóstico de malaria. Biomédica. 2001;21:313-9.

2. Ministerio de Salud. Informe ejecutivo semanal. Malaria en Córdoba: situación epidemiológica y medidas de intervención, Colombia. Inf Quinc Epidemiol Nal. 2001;6:49-64.

3. World Heath Organization. Malaria diagnosis. New perspectives. WHO/MAL/200.1091. Geneva: WHO; 2000.

4. Mason DP, Wongsrichanalai Lin K, Miller RS, Kawamoto F. The panmalarial antigen detected by the ICT malaria $\mathrm{Pf} / \mathrm{Pv}$ immunochromatography test is expressed by Plasmodium malariae. J Clin Microbiol. 2001;39:2035.

5. Win TT, Tantular IS, Pusarawati S, Kerong $\mathbf{H}$, Lin $\mathbf{K}$ Matsuoka $\mathbf{H}$, et al. Detection of Plasmodium ovale by ICT malaria P.f/P.v. immunochromatographic test. Acta Trop. 2001;80:283-4.

6. Tjitra E, Suprianto S, Dyer M, Currie BJ, Anstey NM. Field evaluation of the ICT Malaria P.f/P.v immunochromatographic test for detection of Plasmodium falciparum and Plasmodium vivax in patients with a presumptive clinical diagnosis of malaria in eastern Indonesia. J Clin Microbiol. 1999;37:2412-7.

7. García C, Moreno L, García H. Evaluación de pruebas diagnósticas. En: Moreno L, Cano F, García H, editores. Epidemiología clínica. 3a ed. Atlampa: Editorial Interamericana S.A; 1994.

8. Ministerio de Salud del Perú/Instituto Nacional de Salud del Perú. Evaluación de una prueba rápida inmunocromatográfica por promotores de salud para el diagnóstico de malaria en áreas rurales de la Amazonía peruana, 1999. Informe técnico. Lima: Ministerio de Salud del Perú/Instituto Nacional de Salud del Perú: 1999.

9. Ferro BE, Gonzalez IJ, Carvajal F, Palma GI, Saravia NG. Performance of OptiMAL $\AA$ in the diagnosis of Plasmodium vivax and Plasmodium falciparum infections in a malaria referral center in Colombia. Mem Inst Oswaldo Cruz. 2002;97:731-5.

10. van den Broek I, Hill O, Gordillo F, Angarita B, Hamade $\mathbf{P}$, Counihan $\mathrm{H}$, et al. Evaluation of three rapid tests for diagnosis of $P$. falciparum and $P$. vivax malaria in Colombia. Am J Trop Med Hyg. 2006;75: 1209-15.

11. Wongsrichanalai C, Arevalo I, Laoboonchai A, Yingyuen K, Miller RS, Magill AJ, et al. Rapid diagnostic devices for malaria: field evaluation of a new prototype immunochromatographic assay for the detection of Plasmodium falciparum and non-falciparum Plasmodium. Am J Trop Med Hyg. 2003;69:26-30. 
12. Fernando SD, Karunaweera ND, Fernando WP. Evaluation of a rapid whole blood immunochromatographic assay for the diagnosis of Plasmodium falciparum and Plasmodium vivax malaria. Ceylon Med J. 2004;49:7-11.

13. Playford EG, Walter J. Evaluation of the ICT malaria $\mathrm{Pf} / \mathrm{Pv}$ and the OptiMal rapid diagnostic tests for malaria in febrile returned travellers. J Clin Microbiol. 2002;40:4166-71.

14. Londoño B, Camona J, Blair S. Comparación de los métodos Optimal ${ }^{\circledR}$ y gota gruesa para el diagnóstico de malaria en una zona endémica sin epidemia. Biomédica. 2002;21:466-75.

15. Singh N, Saxena A, Valecha N. Field evaluation of the ICT malaria Pf/Pv immunochromatographic test for diagnosis of Plasmodium falciparum and $P$. vivax infection in forest villages of Chhindwara, central India. Trop Med Int Health. 2000;5:765-70.

16. Cooke AH, Chiodini PL, Doherty T, Moody AH, Ries J, Pinder M. Comparison of a parasite lactate dehydrogenase-based immunochromatographic antigen detection assay (OptiMAL®) with microscopy for the detection of malaria parasites in human blood samples. Am J Trop Med Hyg. 1999;60:173-6.

17. Soto Tarazona A, Solari Zerpa L, Mendoza Requena D, Llanos-Cuentas A, Magill A. Evaluation of the rapid diagnosis test Optimal for diagnosis of malaria due to Plasmodium vivax. Braz J Infect Dis. 2004,8:151-5.

18. Pabón A, Álvarez G, Yánez J, Céspedes C, Rodríguez Y, Restrepo A, et al. Evaluación de la prueba rápida inmunocromatográfica Binax NOW® ICT $P f / P v$ para el diagnóstico de paludismo en un área endémica de Colombia. Biomédica. 2007;27:225-35.

19. Makler MT, Palmer CJ, Age AL. A review of practical techniques for the diagnosis of malaria. Ann Trop Med Parasitol. 1998;92:419-33.

20. World Health Organization. Malaria rapid diagnosis. Making it work. RS/2003/GE/05(PHL). Geneva: WHO; 2003. 